\title{
澳大利亚国家档案馆服务概述及启示
}

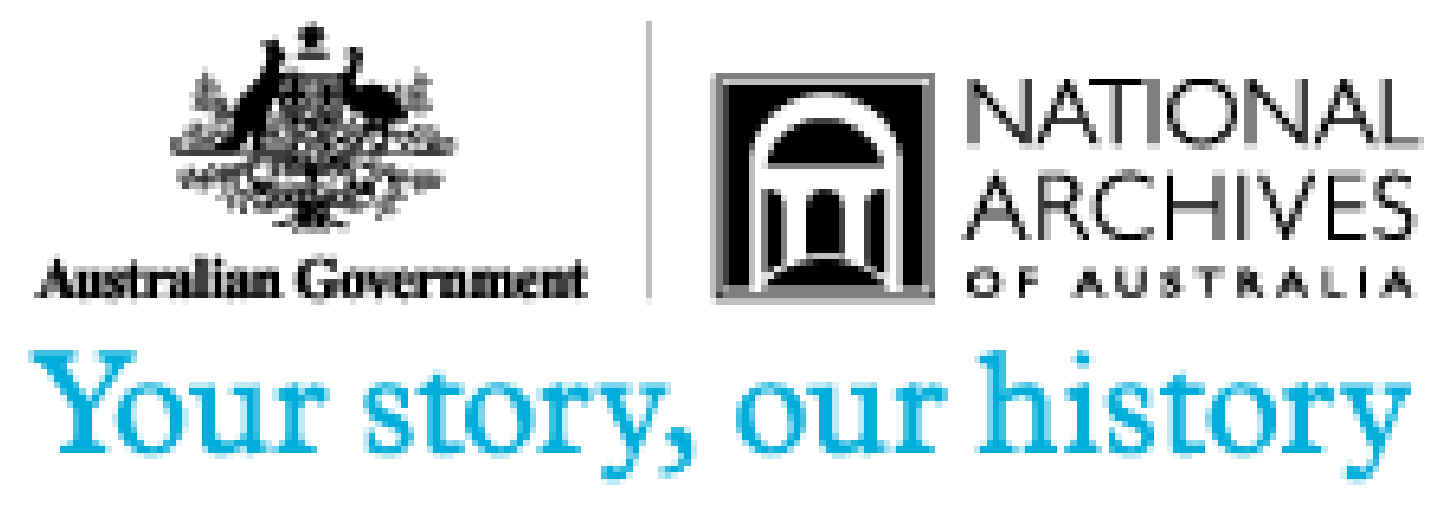

\author{
全国地质资料馆 \\ 资料服务室：高凤琳
}

引用格式请参考: 高风琳.澳大利亚国家档案馆服务概述及启示(V1): 中国地 质调查局发展研究中心, 全国地质资料馆[创建机构]，2020. 全国地质资料馆[传 播机构], 2020-4-20. 10.37088/ngac.ppt2 


\section{$>$ 档案馆服务概述}

> 2020数字连续性政策

$>$ 对地质资料馆藏机构的启示 
澳大利亚国家档案馆（the National Archives of Australia，简称NAA）是国际档案界公认的领 导者，它与国家，地区和国际档案社区广泛合作，学习并分享档案知识和经验。在当今时代，随着 数字技术推动全球变革和创新，NAA 认识到必须转变业务方式，发展数字时代档案馆的作用和能 力，率先提出数字转型政策（Digital transition policy），经过近十年持续推进和发展，奠定了澳 大利亚国家档案馆在数字信息管理方面的领导地位。 


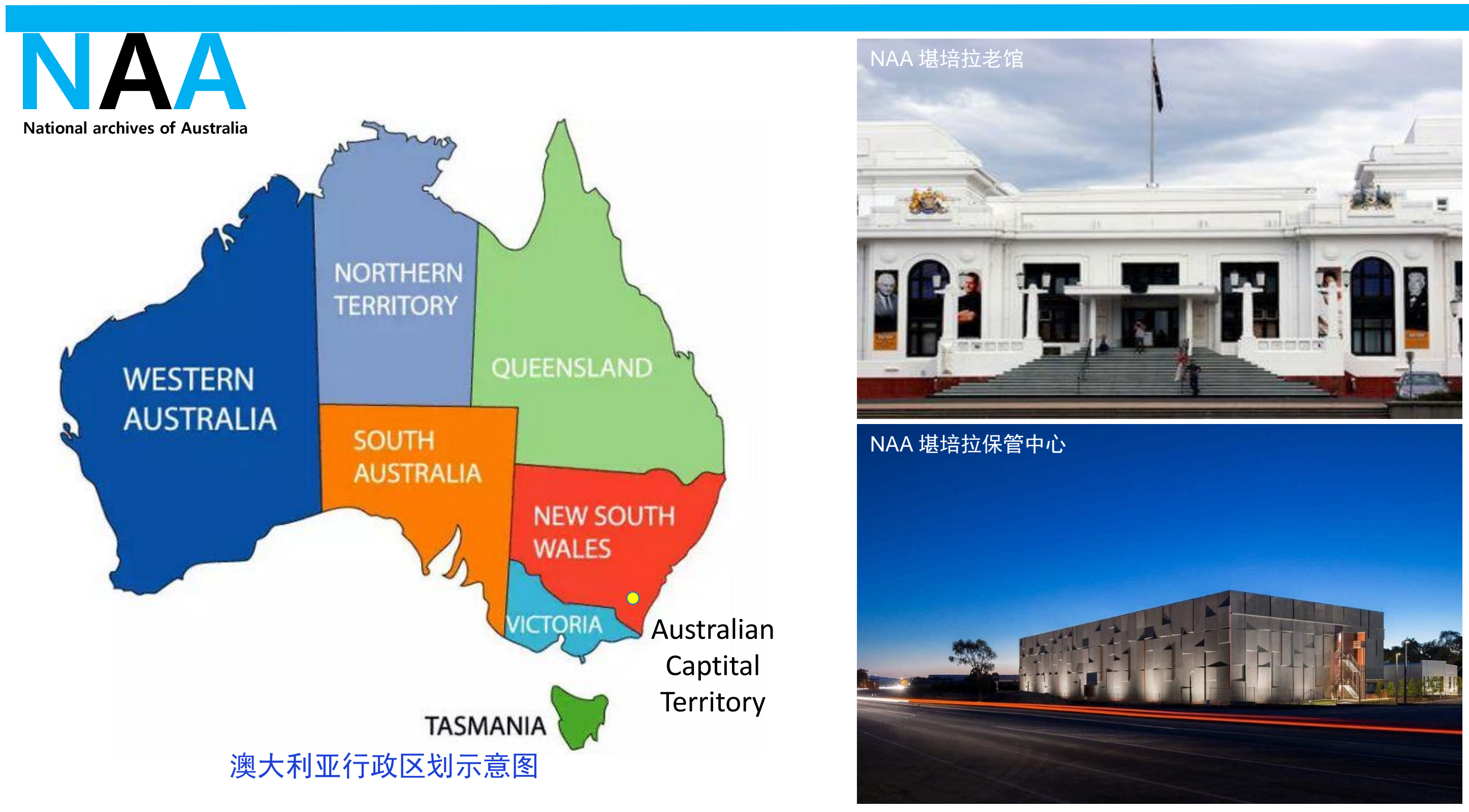




\section{档案馆服务概述一主要职责}

\section{澳大利亚国家档案馆是}

\section{“国家记忆”的保管者}

> 服务对于澳大利亚公民

- 收集、保存、管理和公开澳大利亚政府记录。访问这些记录进行专业研究或者个人兴趣, 以此将澳 大利亚人与其身份和国家历史连接起来

$>$ 服务于澳大利亚政府

- 为从事信息管理工作的澳大利亚政府职员提供指导和服务支撑，设定了数字转换，信息和数据管理 以及记录安全性标准 
- 馆藏数量：超过4000万件记录

- 馆藏来源：主要来自澳大利亚政府部门、法定机构、皇家委员会、军事单位、安全和情报机构、 外交、执法机构以及内阁

- 馆藏主题：移民、兵役、运输、原住民和托雷斯海峡岛民、环境、通讯、安全与情报、外交事务、 艺术、其他主题以及19世纪殖民活动记录

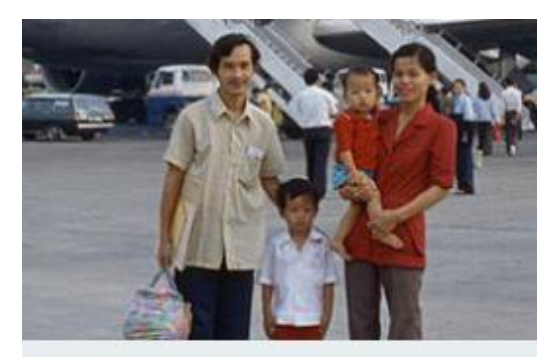

移民与公民身份 $\rightarrow$

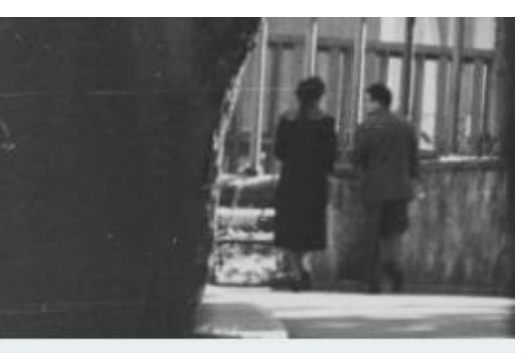

情报与安全 $\rightarrow$

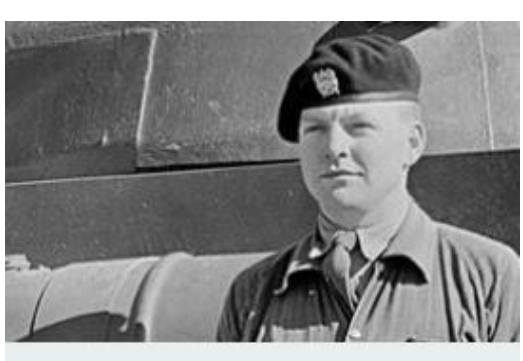

国防和战争服务记录 $\rightarrow$

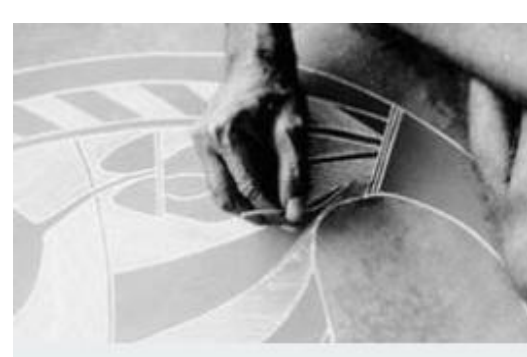

最初的澳大利亚人 $\rightarrow$

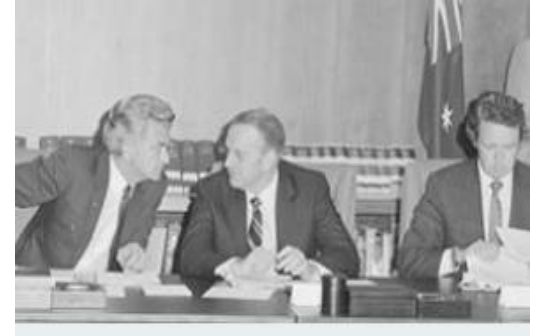

内阁 $\rightarrow$

- 馆藏形式: 纸质、照片、视听记录、录音、地图和计划、海报、物品以及数字等格式 


\section{NAA \\ National archives of Australia}

・服务方式：到馆服务

- 服务内容：展览 演讲

研讨会

原始记录

- 服务对象：所有参观者 研究人员

\section{澳大利亚的间谍活动}

TOURING EXHIBITION

Spy: Espionage in

Australia

๑ Canberra

Revealed for the first time at the National Archives: the curious history of

espionage in Australia. Developed in partnership with ASIO

LEARN MORE

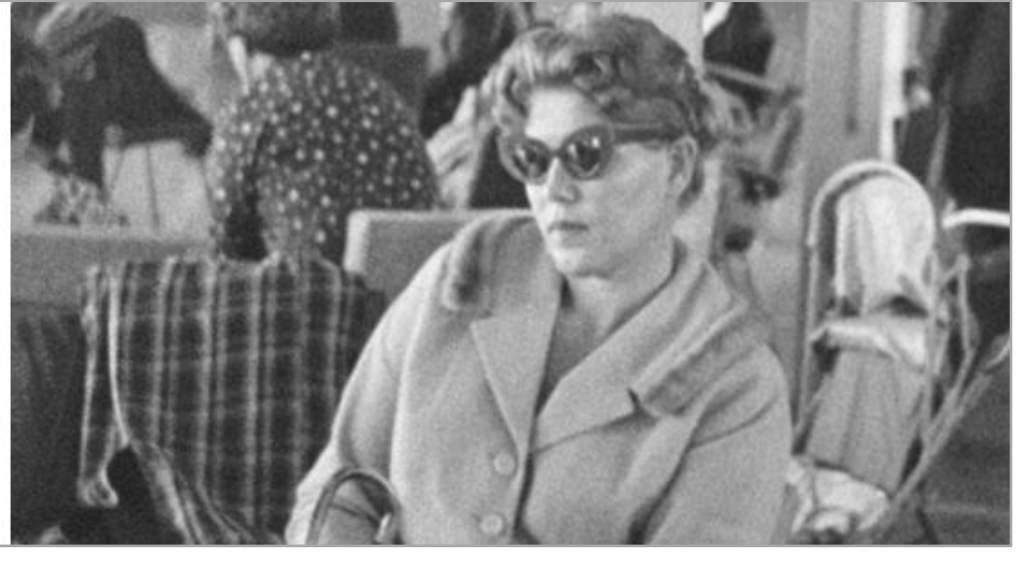

\section{Australian Capital Territory}

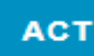

Nsw

NT

QLD

SA

TAS

VIC

WA

\section{Australian Capital Territory}

There is always something exciting to do at our National Office in Canberra.

SHOW MORE $\mathbf{T}$
Address

National Archives National Office Kings Avenue Parkes ACT 2600 Get directions

Hours Mon-Fri Closed

Visitor information $\rightarrow$ $\bullet \equiv$ 


\section{档案馆服务概述一一服务提供}

\section{NAA}

National archives of Australia

- 服务方式: 在线服务

- 服务内容：

汶览

线上展览

检索服务

在线记录申请

数字副本申请

- 服务对象: 所有人

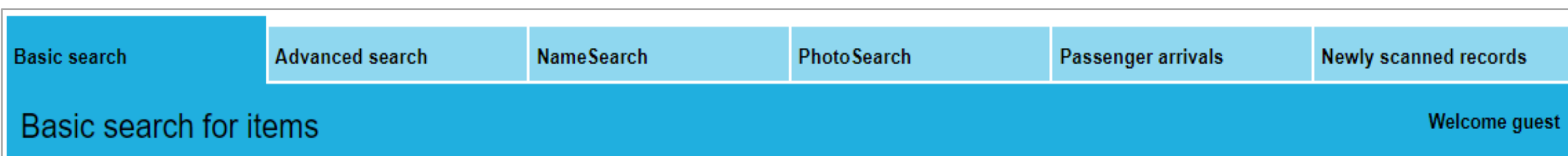

Advanced search for items Bringing Them Home name index
Keywords

\section{Keywords}

Dates
(eg 1921 or 1939 to 1945)

From date To date

\section{Clear}

60,000 个收藏集的描述（系列）

9,000 名创建者和保存者（机构，人员和组织）的详细信息

约800万条记录项目的描述（例如文件，地图，海报)

120万条记录的数字副本

每年数十万的速度向RecordSearch添加项目描述和数字副本
For all up-to-date information on our response to the Covid-19 pandemic, including available services, please check the home page of our website.

The SODA website functionality is now available in RecordSearch, see newly scanned records. Thank you for your continued feedback to improve access to records in the National Archives Aboriginal and Torres Strait Islander people should be aware that this website and the archival collection may contain images, voices or names of deceased persons in photographs, file, audio recordings or printed material.

Material drawn from the archival collection may contain terms that reflect authors' views, or those of the period in which the item was written or recorded, but which may not be considered appropriate today. These views are not necessarily the views of the National Archives of Australia. While information may not reflect current understanding, it is provided in historical context. 
- 服务方式: 到馆和在线服务

- 服务内容：教育资源

- 学校团体参观NAA

- Vroom在线虚拟教室

- 服务对象：学生、教师和学习者
- 全国性竞赛

- ACT学校制宪会议
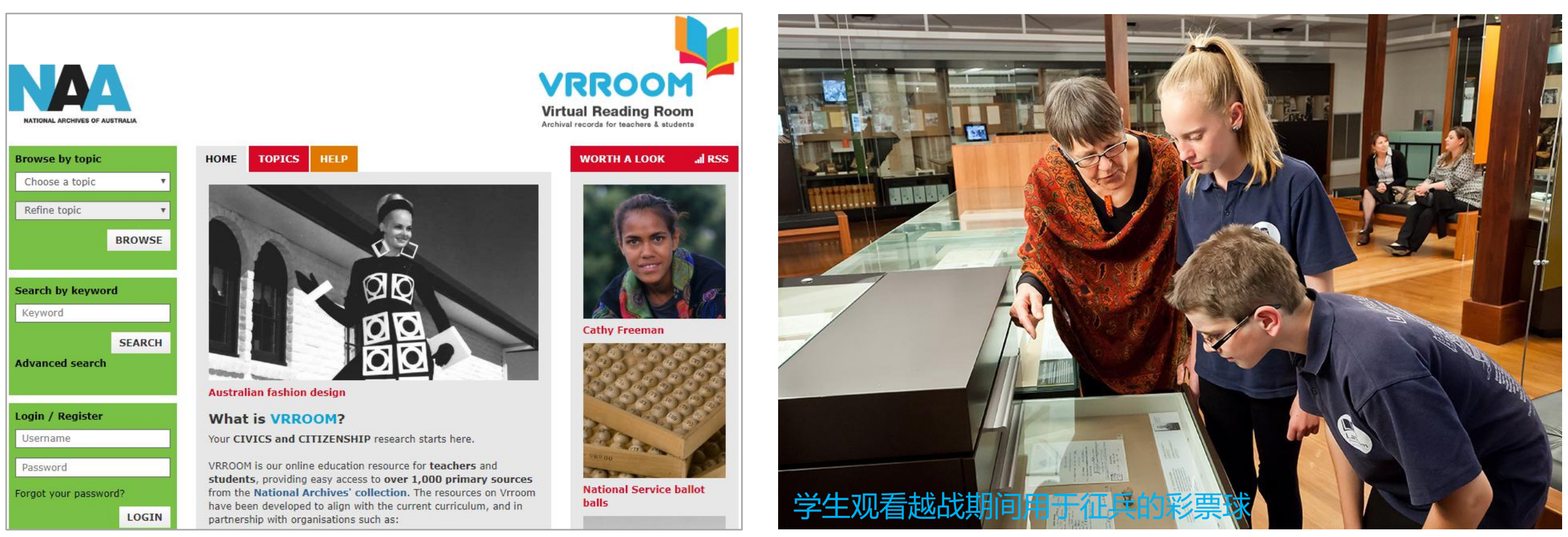


\section{NAA \\ National archives of Australia}

- 服务方式: 电话、邮件

或社交工具

- 服务内容：新闻

图片

演讲

简报

内阁记录

- 服务对象：新闻工作者

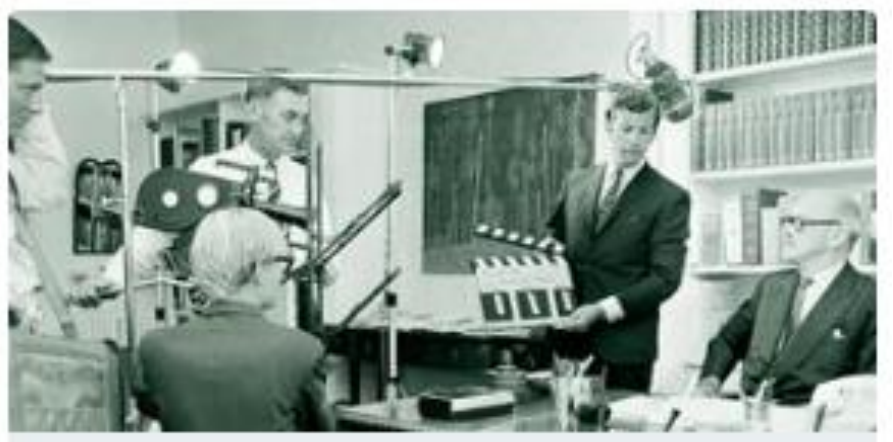

媒体发布 $\rightarrow$

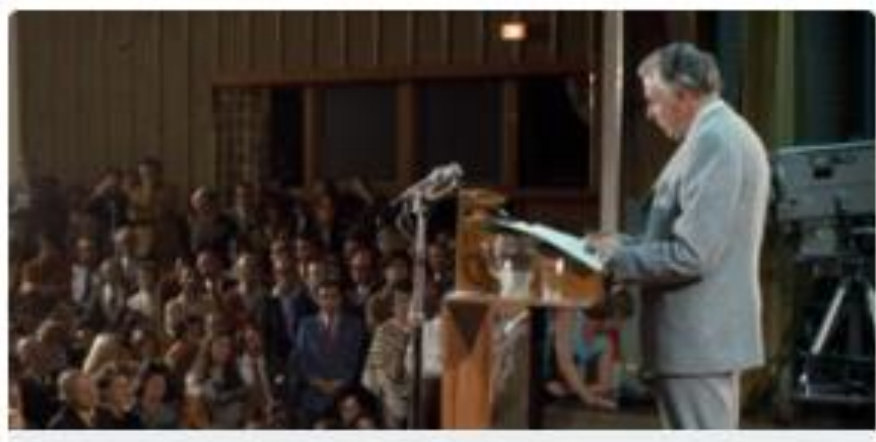

演说 $\rightarrow$

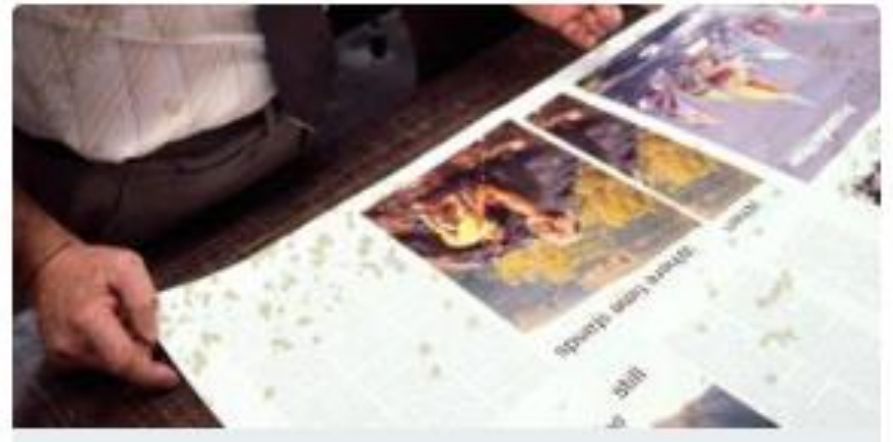

媒体图片 $\rightarrow$

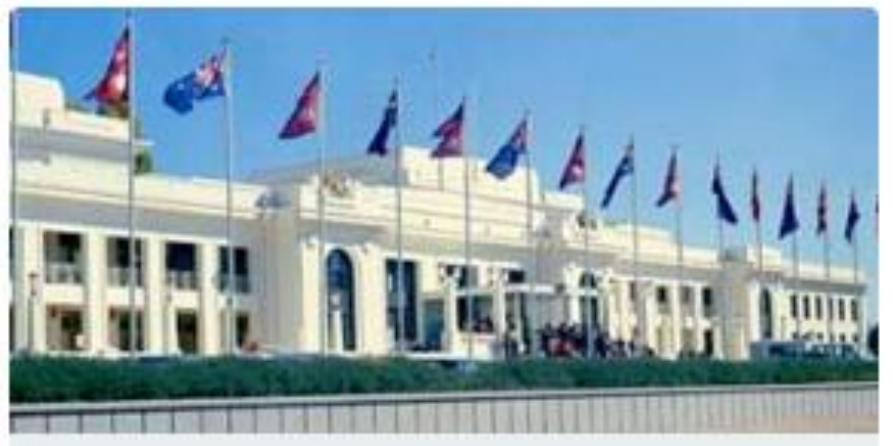

年度内阁记录发布 $\rightarrow$ 
- 服务方式: 在线商店

- 服务内容：图书

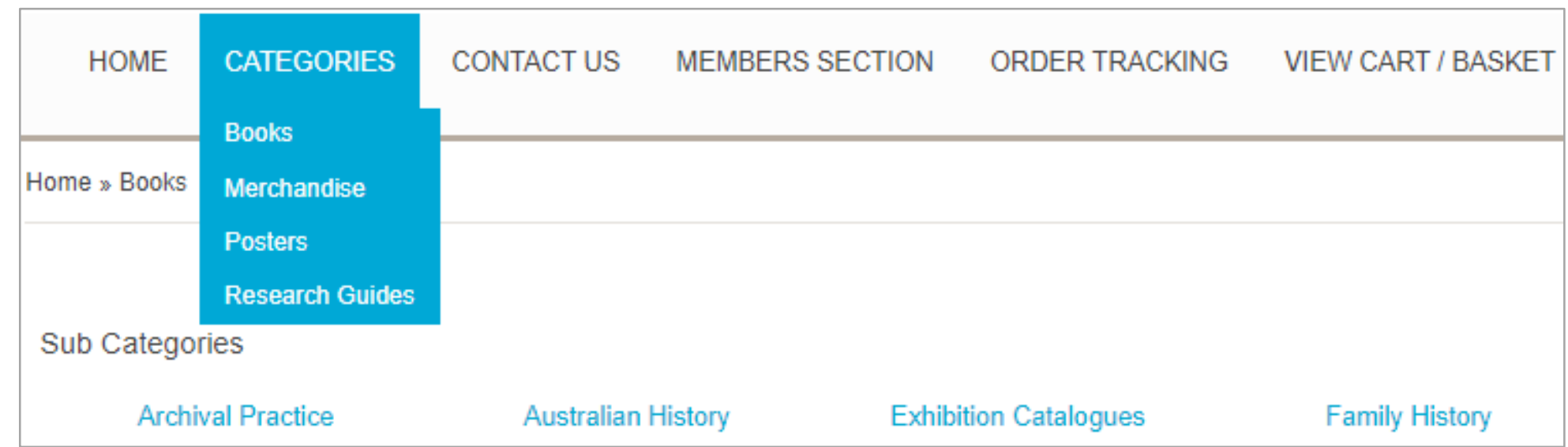

商品

海报

杂志

研究指南

- 服务对象: 所有人

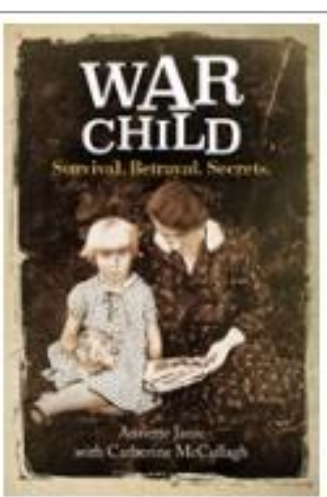

War Child

$\$ 24.95$

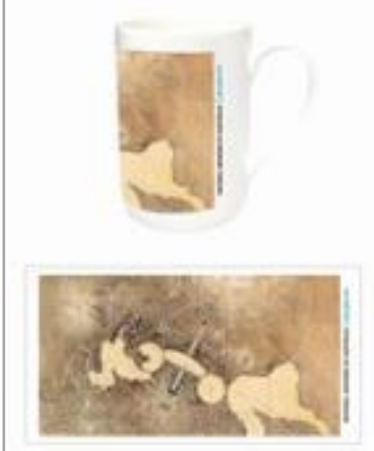

Mug - City and Environs

$\$ 14.95$


Harold Holt

$\$ 4.95$

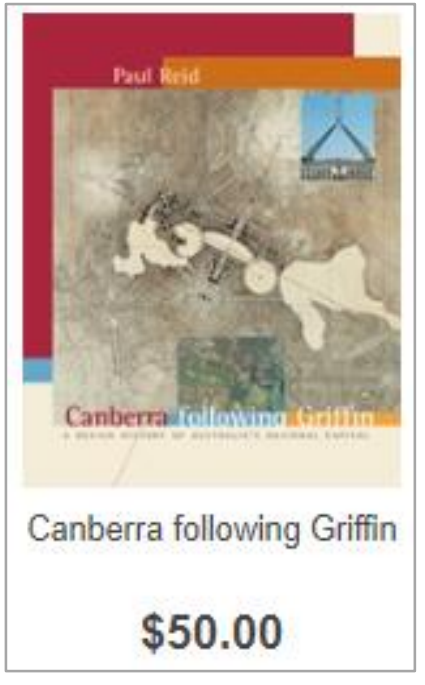




\section{数字连续性政策}

\section{NAA致力于成为数字时代世界领先的档案馆}

2015年10月，澳大利亚国家档案馆发布了2020数字连续性政策（The Digital Continuity 2020)

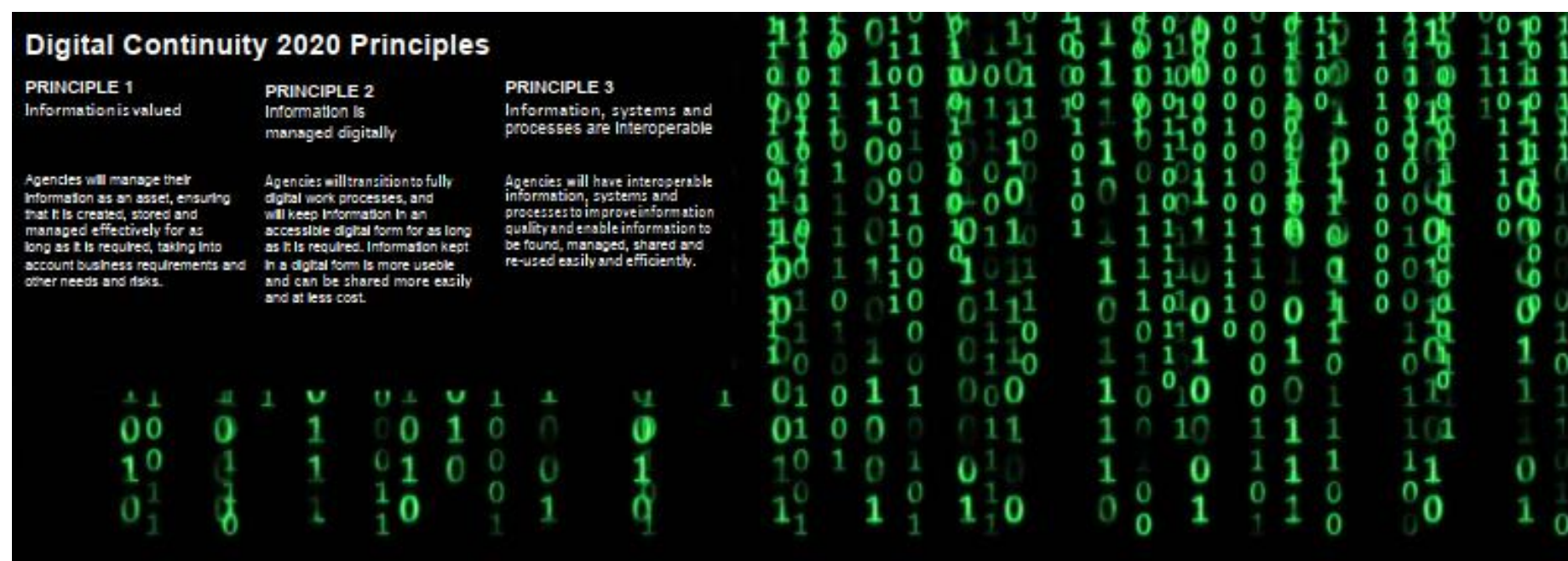




\section{数字连续性政策}

\section{DC2020提出的背景}

- 文件连续体理论

从文件的形成（包含文件管理系统的设计）到文件作为档案保存和利用的全过程管理中连贯一致的 管理方式。该理论打破了文件保管阶段分区，通过构建一个包含4个维度、4大主轴、16个焦点的多维坐 标体系，描述了电子文件生命运动各阶段界限日趋模糊、联系却越发明显的特点，揭示了文件运动在时 间和空间上的连续性和整体性（肖秋会等，2015）

- 数字转型政策 (Digital Transition Policy)

该政策由总理和内阁制定，澳大利亚国家档案馆牵头执行，适用于所有联邦机构，旨在推动澳大利 亚各机构记录逐渐向数字信息和文件管理转型，以提高行政效率，并对各政府机构、NAA，AGIMO等机 构提出具体可行的要求 


\section{DC2020目的}

该政策旨在将强大的数字信息管理集成到所有政府业务流程中

- 优化政府程序和服务的提供

- 使信息为经济和社会利益而重复利用

- 保护澳大利亚人的权益和权利

最终促进整个澳大利亚政府及其内部各机构对信息治理的方法一致。 


\section{数字连续性政策}

\section{DC2020三大原则}

\section{$>$ 原则一：信息是具有价值的，值得重视}

NAA政府信息是重要的战略资产和经济资源，按需求创建、存储和管理信息，并将业务、其他需求和风险的情况 加以考虑，当信息被利用和共享，信息的潜在的未来价值增加

\section{> 原则二: 信息实行数字化管理}

各机构将过渡到完全数字化的工作流程，包括授权和审批在内的业务流程将以数字方式完成，并且信息将以数字 格式创建和管理

\section{$>$ 原则三：信息、系统和过程具可互操作性}

各机构具有可互操作的信息，系统和流程，符合短期和长期管理标准，可提高信息质量，并使信息可以轻松有效 的被发现、管理、共享和重复利用 


\section{数字连续性政策}

\section{NAA 原则一: 信息是具有价值的, 值得重视}

$$
\text { 达成目标一一治理和人员 }
$$

- 机构负责人对其机构的信息治理负责，各机构实施信息治理框架，并每年报告其信 息治理情况

- 信息被有效的创建、存储、使用和管理，如果不再需要，则以可负责的方式销毁

- 机构有适当的政策、过程、标准、控制和量化标指，以确保业务、信息管理和法律 要求得到满足，风险得到管控

- 信息管理人力资源规划和能力发展使各机构能够获得具有专业技能的信息管理人员， 并代表澳大利亚各政府机构履行信息职责 


\section{数字连续性政策}

\section{NA 原则一：信息是具有价值的，值得重视}

National archives of Australia

实施方法——治理和人员

\begin{tabular}{|r|l|c|}
\hline 序号 & \multicolumn{1}{|c|}{ 建议行动 } & 时间 \\
\hline 1 & 年度各机构调查报告 & 每年一次 \\
\hline 2 & 各机按需求管理信息资产; 各机构满足具专业资格或认证的信息管理者的目标 & 2020.12 .31 \\
\hline 3 & 负责信息治理的高级官员个人加入专业协会，以支持他们持续的专业发展。 & 2019.12 .31 \\
\hline 4 & 构建并实施一个信息管理人员的持续发展计划，以进行专业认证 & 2018.12 .31 \\
\hline 5 & 拥有一个首席信息管理官 & 2017.12 .31 \\
\hline 6 & 各机构建立一个信息管理框架 & 2016.12 .31 \\
\hline 7 & 各机构建立一个信息管理委员会 & 2016.06 .30 \\
\hline 8 & 各机构的高级管理人员推动数字信息和文件管理变革，向NAA提交的调查报告经过机构负责人的授权 & 2015.12 .31 \\
\hline
\end{tabular}




\section{数字连续性政策}

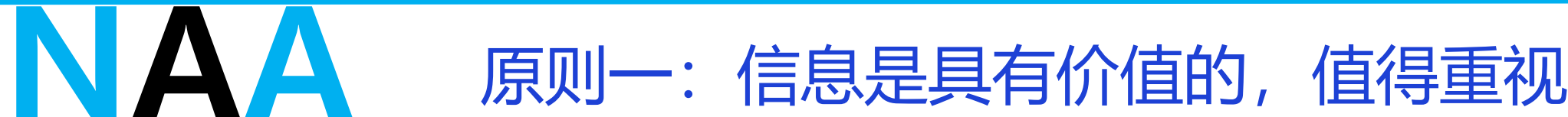

National archives of Australia

\section{澳大利亚国家档案馆的信息治理框架}

\section{原则 人员和责任 系统治理 信息管理策略 信息治理委员会 信息管理标准实施 风险 环境 审核 授权}

所有档案工作人员

所有经理和主任

所有信息技术人员

信息治理团队

信息治理助理总监

首席信息治理官

助理总干事

首席信息官

信息治理委员会

总干事
信息和数据保存在许多系统中

档案管理系统: RecordSearch

行政职能系统：记录保存系统RKS
Archives Act 1983

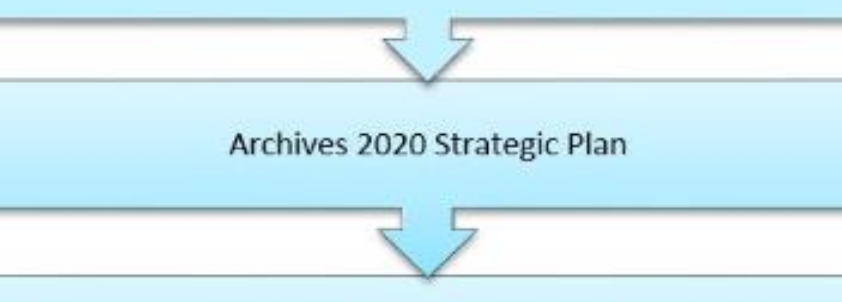

Information and Technology Strategic Direction 2019-22

Information and Data Governance Framework

Archives' policies dealing with information and data assets 


\section{数字连续性政策}

\section{原则二：信息实行数字化管理}

$$
\text { 达成目标一数字资产和流程 }
$$

- 各机构拥有完全数字化的工作流程，只要有需求，以可访问的数字形式保存

- 机构默认以数字方式工作，并将模拟格式或不成熟的数字流程转换为高效、 成熟的数字流程，以实现信息价值最大化

- 对机构业务有益的信息，将模拟格式信息转化成数字格式信息 


\section{数字连续性政策}

\section{原则二：信息实行数字化管理}

\section{实施方法—数字资产和流程}

\begin{tabular}{|c|c|c|}
\hline 序号 & 建议行动 & 时间 \\
\hline 1 & 机构的业务活动、决策和授权都是数字化记录；有价值的机构业务活动，模拟格式信息转化为数字格式 & 2020.12.31 \\
\hline 2 & 各机构实施管理所有信息资产的战略，以支持数字连续性 & 2019.12.31 \\
\hline 3 & $\begin{array}{l}\text { 各机构识别剩余的模拟信息审批流程，并对照档案馆的数字授权框架进行评估，以实现完全的数字授权和 } \\
\text { 工作流程 }\end{array}$ & 2019.06.30 \\
\hline 4 & 各机构识别所有信息资产，评估风险和管理需求，并确定支持数字连续性的策略 & 2018.12.31 \\
\hline 5 & 各机构将大多基于纸张的业务流程转换数字业务流程，并使用数字授权和流程进行常规的决策制定和记录 & 2017.12.31 \\
\hline 6 & 各机构确定高价值和长期的信息资产，评估风险和管理要求，并实施支持数字连续性的战略 & 2016.12.31 \\
\hline 7 & 此日期之后，所有以数字格式创建的文件都以数字方式进行管理 & 2016.01.01 \\
\hline 8 & 各机构减少对纸张的依赖以及对数字和物理格式信息的复制；机构已经确定了基于纸张的业务流程 & 2015.12.31 \\
\hline
\end{tabular}




\title{
数字连续性政策
}

\section{原则三：信息、系统和过程可互操作性}

\author{
达成目标一一元数据和标准
}

- 各机构拥有可互操作的信息、系统和流程，满足短期和长期管理标准，这些标准包 括记录保存的元数据、信息质量以及文件和数据格式的标准

- 机构对业务系统进行信息管理功能评估，以确保信息在需要的时候是可用的，并且 符合业务成果和风险

- 澳大利亚政府流程包含了信息治理需求和规范，以确保信息、系统和服务符合标准， 信息可查找、可共享和可重复利用 


\section{数字连续性政策}

\section{原则三：信息、系统和过程可互操作性}

\section{实施方法一一元数据和标准}

\begin{tabular}{|c|l|c|}
\hline 序号 & \multicolumn{1}{|c|}{ 建议行动 } & 时间 \\
\hline 1 & 信息是基于信息治理和互操作性的格式与元数据标准进行管理的 & 2020.12 .31 \\
\hline 2 & 所有的业务系统都满足信息管理的功能需求 & 2020.12 .31 \\
\hline 3 & 跨部门和整体性政府流程包含了信息治理的要求和规范 & 2020.12 .31 \\
\hline 4 & 所有业务系统根据档案馆业务系统评估框架进行评估，以满足信息管理的功能要求；必要时实现功能需求 & 2018.12 .31 \\
\hline 5 & 所有包含高价值和长期的信息资产的业务系统都需符合最低元数据标准 & 2017.12 .31 \\
\hline 6 & \begin{tabular}{l} 
此日期后，采购的所有业务系统都将达到最低元数据标准，并将根据档案馆业务系统评估框架进行评价， \\
\hline
\end{tabular} & 2016.12 .31 \\
\hline 以满足信息管理的功能要求 & & 200 \\
\hline
\end{tabular}




\section{数字连续性政策}

\section{原则三：信息、系统和过程可互操作性}

National archives of Australia

- 澳大利亚政府信息管理标准

\begin{tabular}{|l|l|}
\hline 序吅 & \multicolumn{1}{|c|}{ 原则 } \\
\hline 原则1 & 系统地管理业务信息 \\
\hline 原则2 & 创建必要业务信息 \\
\hline 原则3 & 充分描述业务信息 \\
\hline 原则4 & 商业信息应适当 \\
\hline 原则5 & 商业信息妥善保管 \\
\hline 原则6 & 商业信息被销毁或转移 \\
\hline 原则7 & 商业信息保存在管理系统 \\
\hline 原则8 & 业务信息可重复利用 \\
\hline
\end{tabular}

信息管理标准

- 记录保存元数据标准

\begin{tabular}{|c|l|}
\hline 当型 & \multicolumn{1}{c|}{ 元素 } \\
\hline \multirow{2}{*}{ 核心 } & 资源标识符 \\
& 创建者 \\
& 创建日期 \\
& 标题 \\
\hline \multirow{3}{*}{ 附加描述 } & 保护标记 \\
& 处置等级 \\
& 格式 \\
\hline & 管理权限 \\
\hline 迁移 & 完整性检查 \\
\hline
\end{tabular}

- 澳大利亚政府定位服务 (AGLS) 元数据标准

\begin{tabular}{|c|c|}
\hline 类型 & 元素 \\
\hline 必要描述 & 创建者、日期、标题 \\
\hline 可选的 & $\begin{array}{l}\text { 受众、可用性、贡献者、 } \\
\text { 领域、授权、关系、权利、 } \\
\text { 来源 }\end{array}$ \\
\hline 推荐 & $\begin{array}{l}\text { 描述、功能、语言、主题、 } \\
\text { 类型 }\end{array}$ \\
\hline 标识符 & 标识符、出版商 \\
\hline
\end{tabular}


1. NAA 的网站主页采用滚动式布局，整体的色彩搭配与 NAA 标志色彩保持一致，采用浅蓝色和白色为主 要背景色，风格简洁明晰，具有较强的标识性。网站主页内容安排主次分明，重点突出，以文字和图片相结合 的方式展现必要信息，提高汶览效率。

地质资料馆藏机构主页面展示内容丰富，应加强对页面布局、形式风格以及色彩搭配方的设计，突出服务 特色、提高网站的汶览效率和可观赏性。

2. NAA服务信息广泛，形式多样，有原始档案记录，数字化副本、到馆和网上展览、演讲、研讨会、媒体 新闻、演讲稿、刊物、在线商店，有针对学生、教师、研究人员、政府机构、商人、志愿者、求职者、新闻工 作者等不同服务对象的专项服务产品，服务范围广泛。

地质资料馆藏机构在资源服务分类上与其相似，而服务对象多集中在专业研究人员，可对服务对象进行拓 展和细化。 
3.澳大利亚国家档案馆将教育启示作为档案馆的一项重要功能加以推广，基于馆藏，推出丰富的教学资源， 采用互动性强的活动，虚拟情景体验、全国性的竞赛、开发在线教学工具或平台，制定校园旅行计划，为教师 和各年龄段的学生提供丰富的教学资源。

地质资料馆藏机构服务资源丰富，建议加大对教育资源服务产品的开发力度，加强馆校合作，推出学生和 教师所需的形式多样的有关地球科学方面的学习资源。

4.澳大利亚的在线数据库RecordSearch功能强大，除了可进行简单搜索和高级搜索外，还提供姓名检索、 照片检索和旅客入境检索，此外还提供最新扫描记录检索，检索功能细化。并通过检索帮助、检索指南、检索 论坛等帮助用户交流检索经验，快速获取信息。

地质资料馆藏机构在线服务资源数量大类型多，增加信息发现和资料查询的帮助或指南等人性化服务，建 立多种形式的咨询服务和交流平台。 
5.数据连续性政策作为一项长期的发展战略，要求所有澳大利亚政府机构推进业务流程的全数字化管理， 我国档案界包括各地质馆藏机构仍然是纸质和电子两种载体并行的双轨制管理模式，随着全球信息化和国家 网络强国战略推进，势必要实现信息全数字化管理的单轨制模式。

地质资料馆藏机构应提高地质信息全数字化管理的意识，为单轨制管理趋势做好政策、法律法规、标准 以及技术方面的准备，逐渐向全数字化流程过渡。 


\section{谢谢大家，欢迎批评指正!}

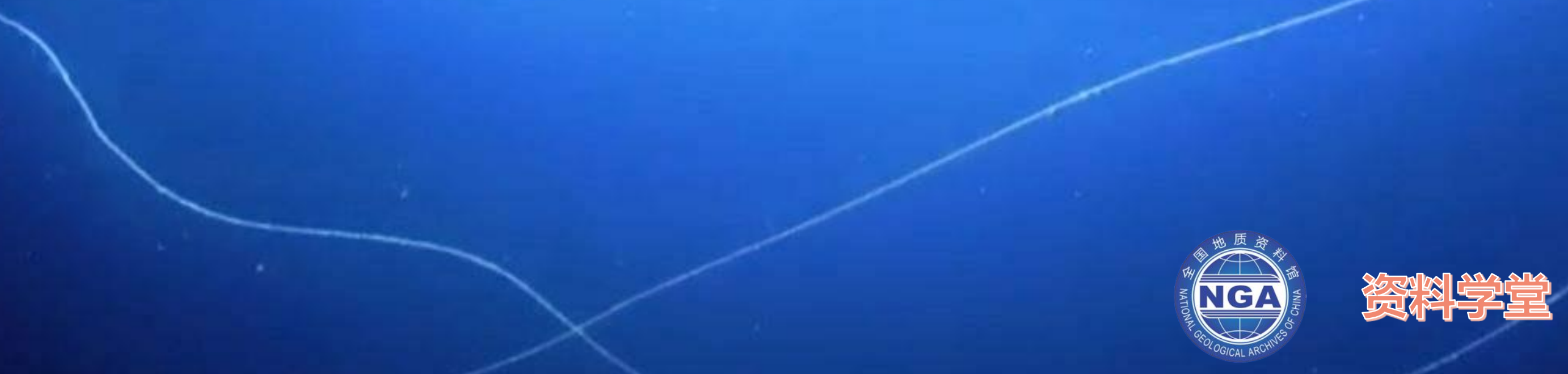

